\title{
EVAR-eaSE: An Easy-to-Use Software for Planning Sac Embolization in EndoVascular Aneurysm Repair Procedure
}

\author{
Laura Cercenelli $\left.{ }^{1, *,+} \mathbb{(}\right)$, Simone Lodi ${ }^{1, \dagger}$, Barbara Bortolani $^{1}$, Chiara Mascoli ${ }^{2}{ }^{(D)}$, \\ Mauro Gargiulo $^{2}$ and Emanuela Marcelli ${ }^{1}$ \\ 1 eDIMES Lab-Laboratory of Bioengineering, Department of Experimental Diagnostic and Specialty \\ Medicine (DIMES), University of Bologna, 40138 Bologna, Italy; simone.lodi8@unibo.it (S.L.); \\ barbara.bortolani@unibo.it (B.B.); emanuela.marcelli@unibo.it (E.M.) \\ 2 Vascular Surgery, Department of Experimental Diagnostic and Specialty Medicine (DIMES), University of \\ Bologna, 40138 Bologna, Italy; chiara.ma@yahoo.it (C.M.); mauro.gargiulo2@unibo.it (M.G.) \\ * Correspondence: laura.cercenelli@unibo.it \\ + These authors contributed equally to this work.
}

Received: 7 July 2020; Accepted: 6 September 2020; Published: 9 September 2020

check for updates

Featured Application: The new software described in the manuscript is intended for application in the vascular field to support the surgeons in preoperative planning of endovascular aneurysm repair (EVAR) procedure.

\begin{abstract}
Background: Endovascular aneurysm repair (EVAR) has become the primary choice of treatment for abdominal aortic aneurysm (AAA) and preventive intraoperative AAA sac embolization (SE) is an effective way to reduce endoleaks after EVAR. Preoperative planning of AAA SE has not been standardized yet, and typically requires a quite long and operator-dependent procedure using standard EVAR planning software. Methods: We introduce 'EVAR-eaSE', a software tool that we have developed to help vascular surgeons to plan the optimal SE procedure during EVAR, providing semi-automatic AAA segmentation and 3D modeling starting from patient scans, and then computing the aneurysm volumes. A preliminary accuracy evaluation on radiological phantoms, and a comparison with the commercial 3Mensio software for EVAR planning are carried out. Results: EVAR-eaSE software showed a good level of accuracy in aneurysm volume reconstruction and calculation (mean difference of $2.5 \%$ between real and segmented phantom volumes). A strong positive correlation $(r=0.940, r=0.954, p<0.001)$ was found between EVAR-eaSE computation of AAA Lumen and AAA Total volumes and the same measurements obtained with 3Mensio, for a database of 26 AAA cases. Conclusions: EVAR-eaSE demonstrated to be an easy-to-use, objective and accurate tool to assist the vascular surgeons in preoperative planning of SE during EVAR.
\end{abstract}

Keywords: aneurysm; EVAR; embolization; 3D modeling; image segmentation; preoperative planning

\section{Introduction}

In the last decade, endovascular aneurysm repair (EVAR) has become the primary choice of treatment for abdominal aortic aneurysm (AAA) in suitable patients due to its lower perioperative morbidity and mortality [1,2].

Endoleaks, i.e., blood flow outside the lumen of the endograft but within the aneurysm sac, are the most frequent causes of reinterventions. Type II endoleaks (ELII), caused by a backflow of collateral arteries into the aneurysm sac, if persistent (ELIIp, $\geq 6$ months), can be associated with an increased incidence of the adverse outcomes, including the sac growth, the reintervention rate, 
the need for conversion to open surgery and AAA ruptures [3-5]. Some authors have suggested routine intra-procedural AAA sac embolization (SE) using different materials (metallic coils, fibrin, or glue) to promote AAA sac thrombosis and to reduce the incidence of ELIIp [6-9]. Previous studies have also shown that the presence of pre-treatment morphological factors, such as multiple patent aortic branch efferent vessels and AAA sac thrombus load, increase the risk of ELIIp after EVAR [4], [10-12]. In particular, a recent study [11] suggested that at least six efferent patent vessels (EPV) and/or a thrombus volume ratio (VR\%, i.e., [AAA thrombus volume/AAA total volume] $* 100$ ) $<40 \%$ of the AAA total volume can identify patients with an increased ELIIp risk, and may lead to a selective prevention with intraoperative SE. Another study [13] investigating for predictors of ELIIp prevention after intraoperative SE with coils during EVAR, identified a Concentration Coil (CCoil = number coils implanted/endoluminal AAA free sac volume) of $0.17 \mathrm{coil} / \mathrm{cc}$ as optimal value to obtain a complete AAA thrombosis.

Currently, the preoperative evaluation of ELIIp risk based on the said morphological features (EPV, VR\%), as well as the choice for the optimal number of coils for SE have not been standardized yet. Aortic aneurysm volumetric estimations, essential for optimal endograft sizing and SE planning, are typically carried by the surgeon using a commercial software for EVAR preoperative sizing and planning (3Mensio ${ }^{\mathrm{TM}}$, Vascular Imaging, Bilthoven, Netherlands, $\left.[14,15]\right)$, but they remain quite operator-dependent and time consuming. Moreover, some procedural steps, such as the estimation of the endoluminal AAA free sac volume available for coils insertion, and the computation of the number of coils to be used for achieving the optimal CCoil, cannot be directly provided by the software.

While in neurovascular field, the SE procedure with coils is more standardized and specific software applications already exist to support surgeon in accurately computing the aneurysm volumes and in predicting the packing density with each coil selection [16,17], for vascular surgery no software tool specifically dedicated to planning SE with coils during EVAR has yet been proposed.

In this paper, the primary aim is to introduce 'EVAR-eaSE', a new software that we have developed to provide the surgeon with an easy, semi-automatic and quick tool to manage the whole process of planning SE during EVAR, including: patient scan segmentation, accurate calculation of aneurysm volumes, input of type and size of the interventional devices (endografts and coils), computation of various parameters useful for supporting the preoperative planning.

After a description of the main functionalities and user interfaces of the new developed software, we present a preliminary evaluation of its accuracy performed on phantoms of known volumes, and a comparison with the commercial 3Mensio EVAR planning software on a retrospective database of 26 AAA cases.

\section{Materials and Methods}

\subsection{EVAR-eaSE Description}

The EVAR-eaSE software was developed in MATLAB (version R2019a, The MathWorks, Inc., Natick, Massachusetts, United States) a technical computing language used mostly for high-performance numeric calculations and visualization, that provides many thematic libraries, including image processing tools, and easy implementable Graphic User Interface (GUI) mechanisms.

EVAR-eaSE is composed of three processing modules and corresponding GUI:

1. Upload Window (UW): handles the uploading of DICOM (Digital Imaging and Communications in Medicine) files, e.g., computed tomography angiography (CTA) scans, the image pre-processing, the visualization of CTA volume rendering, and the selection of a region of interest (ROI).

2. Segmentation Window (SW): shows a contrast-enhanced version of the ROI selected in the previous window, and provides the ROI segmentation both for the pervious (lumen) and thrombotic part of the AAA; segmentation results are displayed in real time as contours on multi-planar volume reconstruction. 
3. Display and Computation Window (DCW): displays the 3D models resulting from AAA segmentation and provides measurements useful for planning, including the optimal number of coils for SE.

The functions of each module are summarized in the framework reported in Figure 1, and are described more in detail in the following paragraphs.

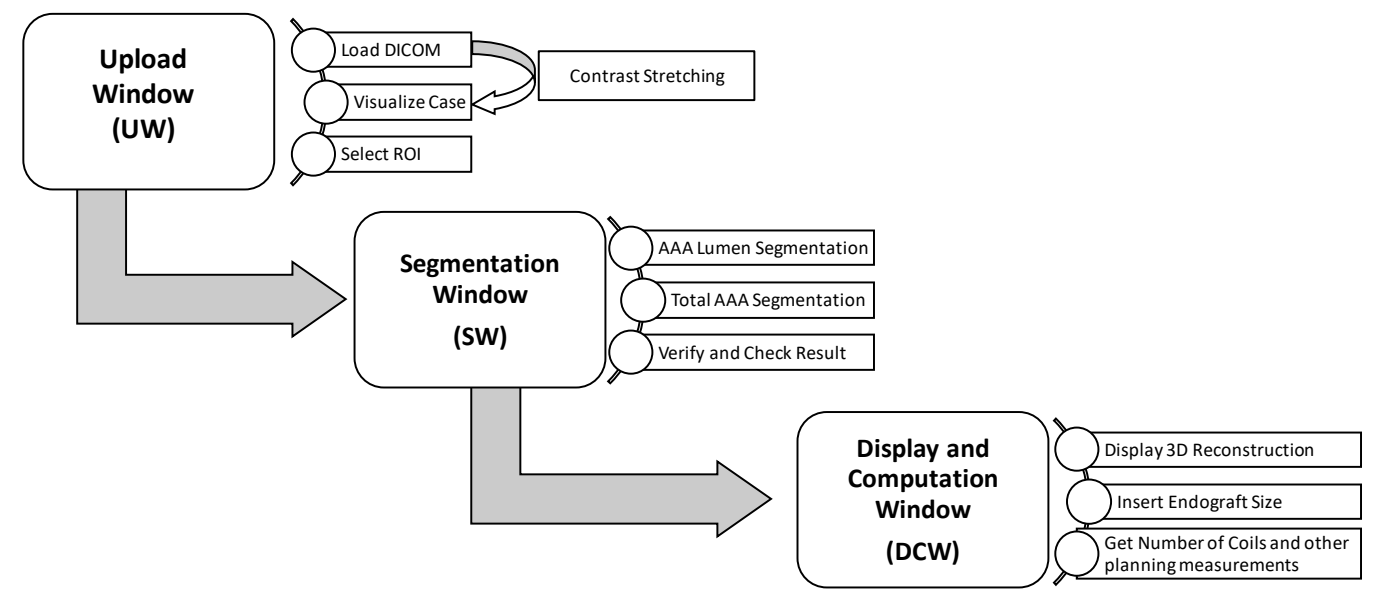

Figure 1. EVAR-eaSE framework: the three interconnected processing modules (Upload Window-UW, Segmentation Window-SW, Display and Computation Window-DCW) and the main software functions implemented in each module.

\subsubsection{Upload Window (UW)}

Upload Window (UW) essentially allows to easily import DICOM files and select a ROI in the image for further segmentation. The developed GUI for UW is depicted in Figure 2.

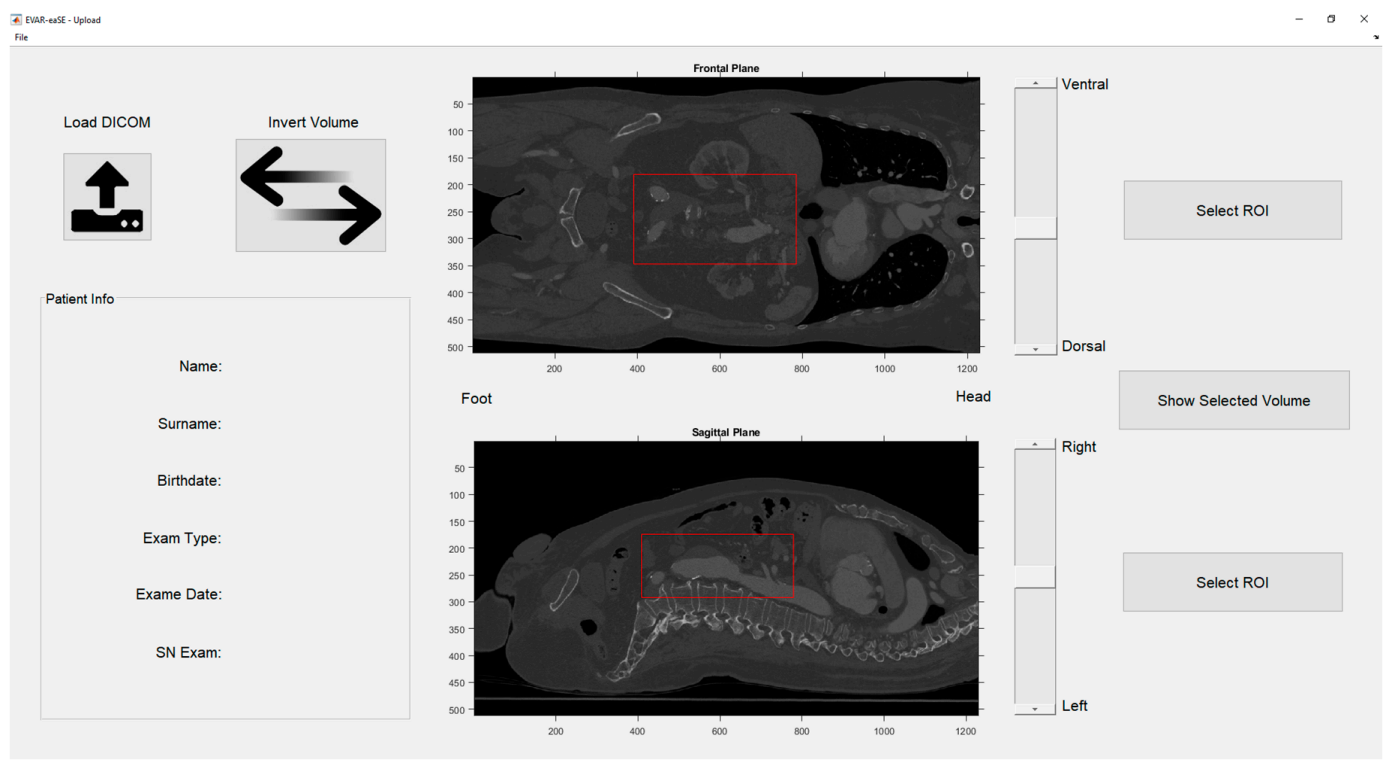

Figure 2. Graphical user interface of Upload Window (UW).

a. UW functions

Load DICOM (square button with an arrow icon, in the top left): this function allows the operator to upload a DICOM file folder, automatically managing some possible issues related to file selection, such as the presence in the same folder of various scans even coming from different diagnostic 
modalities, or external files not relative to the case under examination. To address these possible issues, all available image scans are shown to the user in a preliminary window, in which each folder has a description. The user can select the folder to load, simply by clicking on it. During loading, the DICOM files with a reduced number of slices and/or with a too large slice thickness (low resolution) than the other files in the same folder, are recognized by the software and excluded.

Invert Volume (rectangular button with two-arrows icon, in the top left): this command is used to mirror the imported DICOM scans, in order to switch between "head-foot" and "foot-head" orientation. This may be useful to set the right orientation ensuring that in axial plane of the Segmentation Window, when scrolling the slider up the scan advances towards the head, when scrolling down it goes towards the foot.

Select ROI (rectangular buttons in the right side): it allows for selection of a ROI, both in the frontal and sagittal planes, by clicking and dragging a rectangular selection on the image. Once the ROI is selected in both planes, the central button (Show Selected Volume) becomes active.

Show Selected Volume (central button in the right side): when pressed, it opens the SW that provides the segmentation only for that portion of the image resulting from the intersection of the selected ROI in the two planes.

File menu: contains Save and Import functions to save the current EVAR-eaSE working file and import a previously created and saved file, respectively.

\section{b. UW outputs}

Patient Info (box in the bottom left): in this section the patient information and exam data (e.g., exam type, exam date) are displayed as soon as the DICOM files are loaded.

Image frames (in the central part of the window): the loaded image scans are displayed along two planes: frontal (or coronal) and sagittal. The scans can be scrolled along ventral-dorsal (for frontal plane) and left-to-right (for sagittal plane) directions, using the nearby sliders.

\subsubsection{Segmentation Window (SW)}

In the Segmentation Window (SW), the user can segment in a quite semi-automatic way both the pervious part of the aneurysm (AAA lumen) and the AAA total volume, including also the thrombotic formation. The segmentation results are then displayed on multi-planar views (frontal, sagittal and axial planes). The GUI for SW is depicted in Figure 3.

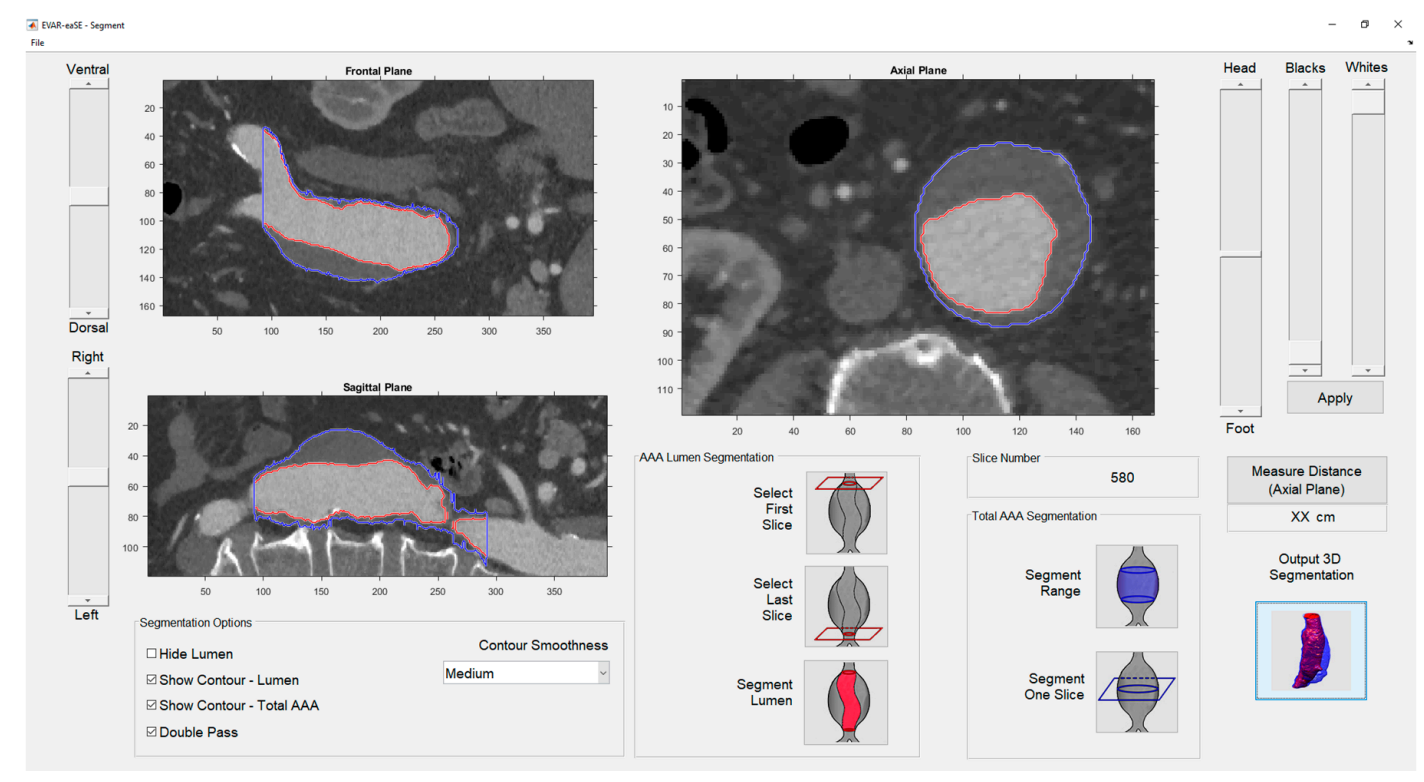

Figure 3. Graphical user interface of Segmentation Window (SW). 


\section{a. SW functions}

Black and White sliders (in the upper right part of the window): preliminary custom image processing on the selected ROI may be performed using these sliders that allow the user to change the image contrast, working independently on dark (Black) and bright (White) pixels. Once done, the operator can confirm the selected contrast level by pressing the Apply button.

Measure Distance (rectangular button under Black and White sliders): pressing this button a two-points ruler can be drawn in the active slice. After placing the points, the distance between them is calculated and displayed in centimeters. This function can be used only in the axial plane, and when it is activated the segmentation contours are temporarily hidden from the axial view.

Segmentation In the center and bottom right of the window are present two segmentation modules developed for an efficient and quick segmentation of both the AAA lumen (AAA Lumen Segmentation) and the entire vessel with aneurysm (Total AAA Segmentation), while in the bottom left there is a panel for adjusting the segmentation parameters (Segmentation Options).

\section{AAA Lumen Segmentation}

Select First Slice (button with a red square in the top): allows the user to select and segment the first slice of the vessel lumen just under the renal arteries, in the axial view. The software detects automatically the right contour when the user draws manually near the lumen area.

Select Last Slice (button with a red square in the bottom): similar to the previous one, allows the user to select and segment the last slice of the vessel lumen just under the iliac bifurcation. The software detects automatically the right contour when the user draws manually near the lumen area.

Segment Lumen (button with a highlighted red lumen): this function will start the segmentation of the AAA lumen, considering the volume between the selected first and last slices. The segmentation process is automatic, making use of the Active contours MATLAB function, in particular the Chan-Vese variant. Active contours segmentation algorithm (also called snakes) is an automatic, iterative method where you mark locations in the image by drawing regions, called a seed mask. Active contours grows (or shrinks) these seed shapes to fill the borders of the region in the image. In particular, we used the Chan-Vese variant, which was first designed by Tony Chan and Luminita Vese to segment objects without clearly defined boundaries [18]. The Active Contours algorithm was chosen since, as previously reported [19], it is a good option for segmenting images that have varying intensities, low contrast boundaries and noises; moreover, the exact vessel segmentation is not possible by separating vessel and bone regions through only thresholding or region-based approaches [20].

In our specific case, the software continuously evaluates the centroid of the current detected region and prolongs it to the next slice in order to use it as the starting seed of the new segmentation. In this way, the segmentation process can be completed without requiring other inputs from the user. Since the aorta is quite a cylindrical volume with very little askew sideways, the algorithm works efficiently once the first slice has been correctly segmented. The result of the automatic segmentation can be visually checked by the operator, and overall refinements may be added by manual segmentation.

\section{Total AAA Segmentation}

Segment Range (button with a blue highlighted portion): by pressing this button, the user can start the segmentation of the entire AAA, i.e., the volume between the selected first and last slices, also including the thrombus part. The segmentation process is based on the same MATLAB functions used for segmenting the AAA lumen. In the axial view, a contour must be drawn around the outer limit of the artery, starting from the first slice. The user has to scroll along the volume of 10-12 slices using the slider next to the axial view panel, and then to repeat the drawing process. The recommended value of 10-12 slices may guarantee a quite fast and accurate segmentation result. 
Segment One Slice (button with a blue square in the middle): if pressed, the segmentation is done only on the slice visible in the axial window. This option is useful for adjusting errors that may result from the Segment Range function.

\section{Segmentation Options}

Hide Lumen (first checkbox in the top left side of the panel): this box, which becomes active only after the AAA lumen segmentation, places a "patch" (a piece of texture) on the already processed lumen and activates a specific image contrast to make it easier for the user the selection of AAA contours for Total AAA segmentation. It helps also to identify in the image the EPV of the aneurysm.

Show Contour Lumen (second checkbox from the top in the left side of the panel): activates/deactivates the red outline of the AAA lumen after it is segmented. It acts on all the views.

Show Contour Total Vessel (third checkbox from the top in the left side of the panel): activates/deactivates the blue outline of the thrombotic part of the total AAA after it is segmented. It acts on all the views.

Double Pass (last checkbox in the bottom left side of the panel): activates/deactivates a double pass (i.e., head-foot and foot-head) analysis, which repeats the segmentation process for both directions and then merges the results, in order to avoid underestimation of the segmented volume. It is recommended to leave it on and, in case of errors, modify the contour smoothness.

Contour Smoothness (pop-up menu, in the right side of the panel): five step menu-none, low, medium, high, max-which modifies the regularity of the contour during the segmentation. Changing the contour smoothness step from "none" to "max" the obtained effect is a gradual increasing in the smoothing of the segmented region profile. Higher values are safer in order to not include unwanted structures (e.g., the collateral small vessels that should be excluded), while lower values are prone to guarantee more adherence to the real silhouette of the segmented anatomical structure. The default value is set to "medium" which is considered and tested as a fair compromise; however, the user can try with different smoothness levels if during the visual checking of results, he observes under- or over-estimated segmented regions.

\section{b. SW outputs}

Three image frames (in the main part of the window): the selected volume is displayed along three planes: frontal and sagittal, on the left side, and axial, in the center. In each plane the volume can be scrolled along ventral-dorsal (for frontal plane), left-to-right (for sagittal plane) and head-to-foot (for axial plane) directions, using the nearby sliders. In the image frames, the segmentation results are displayed as multi-planar contours reconstruction: red contours identify the segmented AAA lumen, the blue ones the segmented total AAA including thrombus. The contours are overlaid in all the planes and they can be activated/deactivated by the Show Contour Lumen and Show Contour Total AAA checkboxes.

Slice Number (under the axial plane frame, on the right): indicates the slice number, relatively to the entire volume. It is useful for having a reference of the processed slice with respect to the original scan.

Output 3D Segmentation (button with icon of the segmented volumes, in the bottom right side): if pressed, the user can move to the final window (Display and Computation Window-DCW) where the output 3D segmented volumes are displayed in large size.

\subsubsection{Display and Computation Window (DCW)}

In this panel, the segmented 3D models are displayed, and the output measurements are provided. The GUI for DCW is depicted in Figure 4. 


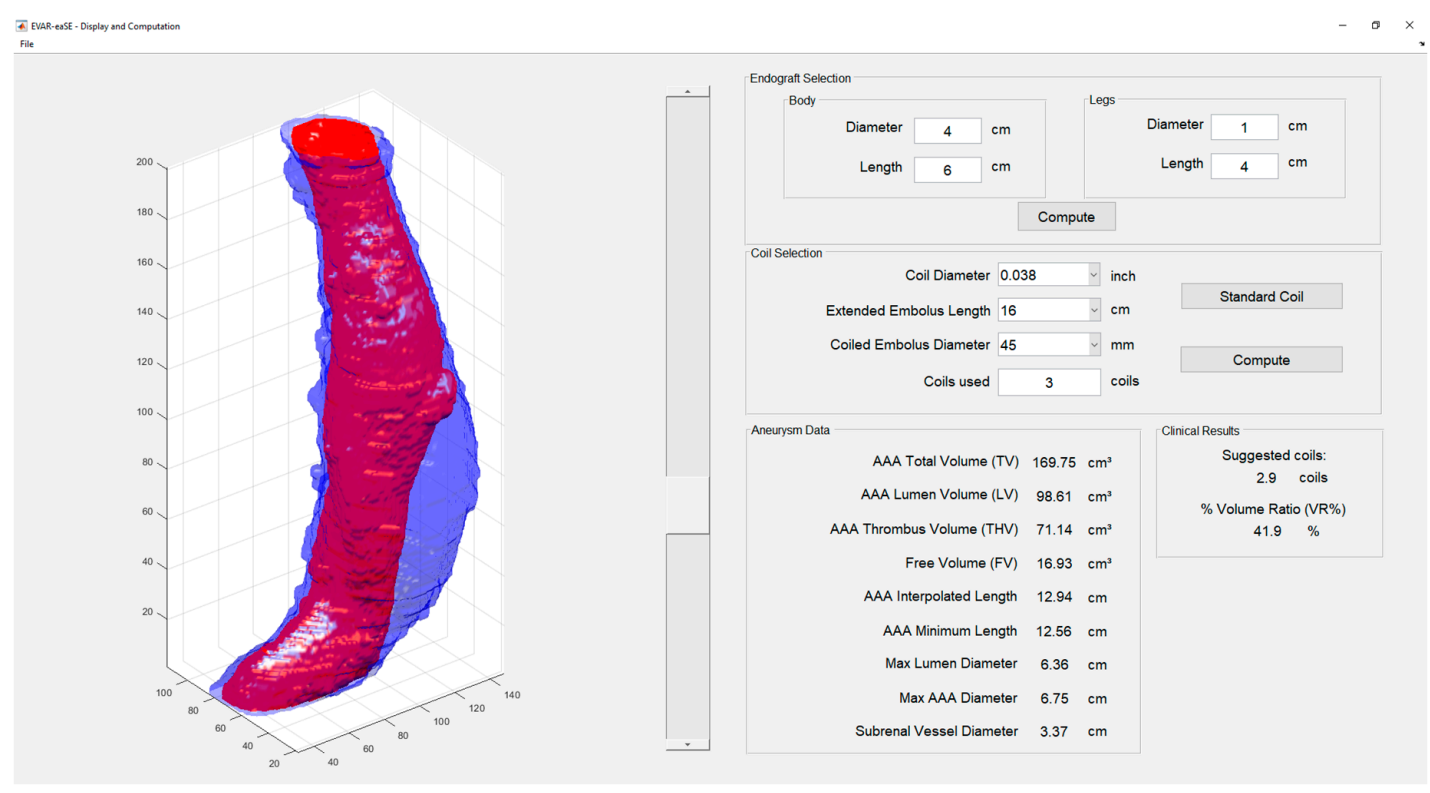

Figure 4. Graphical user interface of Display and Computation Window (DCW).

\section{a. CW functions}

In the left side of the window, the segmented 3D models are displayed, including both the lumen in red, and the total AAA in blue. The transparency of the external total AAA volume can be regulated via the nearby slider.

Endograft Selection (top right part of the window): the user can set the endograft size, including its dimensions (diameter and length) in the appropriate fields. Pressing the Compute button, the endoluminal Free Volume (FV) available for SE with coils is computed as difference between the AAA-Lumen Volume (LV) directly derived from image segmentation, and the estimated endograft volume. The latter is approximated with three cylinders: the main body and the two side legs. For the main body the total inserted length is taken into account, while for side legs only their portion between the main body end and the iliac bifurcation (carrefour) was considered in order to calculate the effective volume occupied by the endograft in the aneurysm sac (Figure 5).

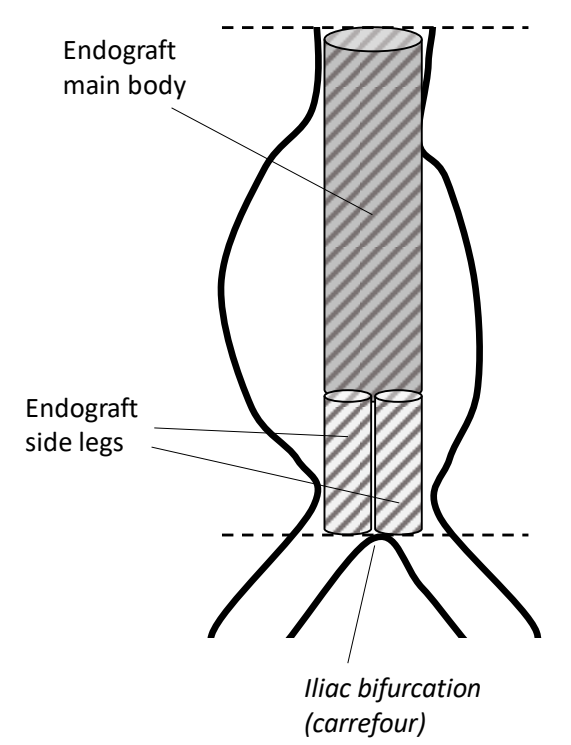

Figure 5. Schematic representation of the estimated endograft volume (the dotted cylindrical shapes). 
Coil Selection and Computation (middle right part of the window): the user can use the pop-up menu to select a specific coil model and size (diameter, length, and coiled embolus diameter) or press the Select Standard Coil button to select a standard coil type (namely the Cook Medical MReye IMWCE-38-16-45 coil). By pressing the Compute button, the software calculates the optimal number of coils required for SE, based upon the previously evaluated FV. The number of coils is obtained as FV multiplied by the optimal coil concentration (CCoil) of $0.17 \mathrm{coils} / \mathrm{cm}^{3}$, previously reported by Mascoli et al. [13].

\section{b. CW outputs}

The various output measurements computed by the software tool are displayed in DCW. A brief description of each measurement is reported in Table 1.

Table 1. Output measurements provided by the EVAR-eaSE software. AAA = abdominal aortic aneurysm.

\begin{tabular}{|c|c|}
\hline Measurement & Description \\
\hline AAA-Total Volume (TV) & $\begin{array}{l}\text { Volume (in } \mathrm{cm}^{3} \text { ) computed for the segmented total AAA, also } \\
\text { including the thrombus. }\end{array}$ \\
\hline AAA-Lumen Volume (LV) & Volume (in $\mathrm{cm}^{3}$ ) computed for the segmented AAA lumen. \\
\hline AAA-Thrombus Volume (THV) & $\begin{array}{c}\text { Volume (in } \mathrm{cm}^{3} \text { ) computed for the segmented thrombus, } \\
\text { calculated as difference between AAA-Total Volume (TV) and } \\
\text { AAA-Lumen Volume (LV). }\end{array}$ \\
\hline \% Volume Ratio (\%VR) & $\begin{array}{l}\text { Percentual volumetric ratio between the thrombus volume } \\
(\mathrm{THV}) \text { and the total AAA volume, calculated as }(\mathrm{THV} / \mathrm{TV}){ }^{*} 100 \text {. }\end{array}$ \\
\hline Free Volume (FV) & $\begin{array}{c}\text { Volume (in } \mathrm{cm}^{3} \text { ) computed as the difference between the LV and } \\
\text { the estimated endograft volume in the AAA }\end{array}$ \\
\hline AAA Axis Length & $\begin{array}{l}\text { Center line length, i.e., the line passing by the centroid of the } \\
\text { AAA along each slice }\end{array}$ \\
\hline Minimum AAA Length & Straight distance between the first and the last selected slices \\
\hline Max Lumen Diameter & Maximum diameter for the segmented AAA lumen \\
\hline Max AAA Diameter & Maximum diameter for the segmented entire AAA. \\
\hline Subrenal Vessel Diameter & $\begin{array}{l}\text { Diameter of the aorta just under the renal arteries, calculated as } \\
\text { mean aorta diameter in the first five slices of the vessel, starting } \\
\text { from the first selected slice. }\end{array}$ \\
\hline
\end{tabular}

Number of coils

The computed optimal number of coils, starting from the computed FV.

An example of EVAR-eaSE application to a real patient AAA case is shown in the Supplementary Video Material (Video S1).

\subsection{EVAR-eaSE Evaluation}

In this section, we provide results of the evaluation we performed for the developed software. First, a preliminary assessment of EVAR-eaSE accuracy in segmentation and volumetric computation was carried out using solid plastic phantoms of known volumes, typically used in radiology. Then, we applied the new software to a retrospective database of AAA cases treated with coil embolization, and we compared the obtained volumetric results with the AAA volumes estimated with the commercial planning software 3Mensio.

\subsubsection{Phantom Tests}

Two CT imaging phantoms, i.e., two solid plastic models of regular shape and geometry responding in a similar manner to human tissues under CT, were provided by the Medical Physics Unit of S. Orsola Hospital, University of Bologna (Figure 6). 
(a)

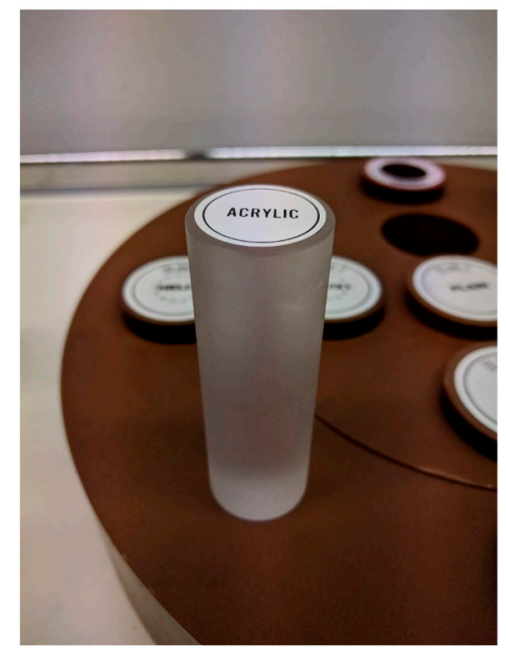

(b)

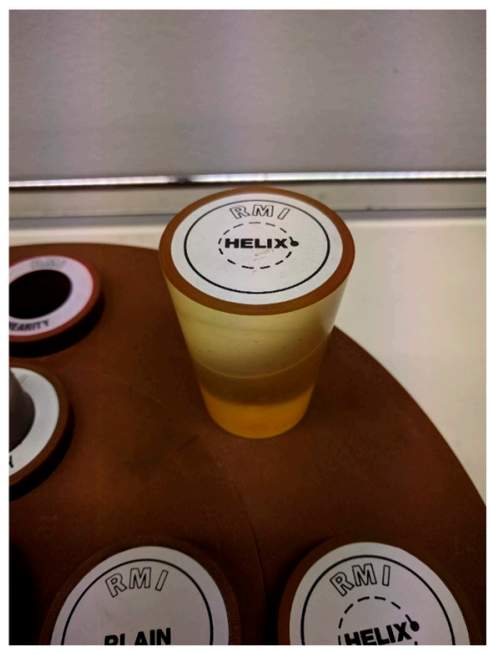

Figure 6. The two Computed Tomography (CT) imaging phantoms, one made of acrylic material (a), the other of Lucite and with helix shape (b), used to evaluate the segmentation accuracy of the EVAR-eaSE software.

The first (Phantom 1), made of acrylic material and having a cylindrical shape, was chosen to simulate a highly-contrasted medium, like the vessel after the infusion of the contrast medium. The second (Phantom 2) was a "helix phantom" made of Lucite and with a truncated cone shape with lead markers inserted according to a helicoidal pattern. This phantom was selected in order to take into account the effect of possible artifacts from metal objects, like the implanted endograft in the aneurysm. For the two phantoms, the size was measured with a precision gauge, then the volume was assessed ( $54.29 \mathrm{~cm}^{3}$ and $110.33 \mathrm{~cm}^{3}$ for Phantoms 1 and 2, respectively). CT scans of the two phantoms were performed varying the acquisition parameters, i.e., the slice thickness $(0.67 \mathrm{~mm}, 2 \mathrm{~mm})$ and the pitch ratio, PR, ( 1 = no slide overlap, 0.5 = slice overlap $)$.

\subsubsection{Comparative Analysis with 3Mensio}

For comparative analysis, we conducted a retrospective study on 26 AAA cases treated with coil SE and planned with 3Mensio software currently used by vascular surgeons at S. Orsola Hospital in Bologna.

We started from the CTA scan of each case and we proceeded with EVAR-eaSE segmentation of the AAA-LV and AAA-TV, and the further calculation of all the output measurements reported in Table 1 . Then, the EVAR-eaSE volumes were compared with those previously obtained with 3Mensio planning software.

\subsubsection{Data Analysis and Statistics}

In the phantom tests, the acquired CT scans were processed using the EVAR-eaSE software and the Mean output volume (Mean "computed" volume) was calculated and compared with the corresponding measurement taken with the gauge ("real" volume) on each phantom. To evaluate if the different $\mathrm{CT}$ acquisition parameters may have influence on the volumes obtained by EVAR-eaSE processing, the independent samples t-test was used. Finally, one sample t-test was used to determine whether the Mean "computed" volume for each phantom was statistically different from the "real" volume measured with gauge. A $p$ level of 0.05 was chosen.

In the comparative analysis, Means and SD were calculated for AAA-LV and AAA-TV by averaging values obtained over the 26 cases with both the EVAR-eaSE application and the previously performed 3Mensio planning, then paired samples correlation was evaluated. 
Statistical analyses were performed in SPSS (IBM SPSS, Armonk, New York, NY, USA).

\section{Results}

\subsection{Results of Phantom Tests}

For both phantoms, the different CT scan parameters (i.e., slice thickness and PR) do not significantly change the volumetric results provided by the software (Tables 2 and 3).

Table 2. Results from preliminary assessment of EVAR-eaSE accuracy using radiological phantom 1.

\begin{tabular}{|c|c|c|}
\hline Phantom 1 & $\begin{array}{c}\text { Slice Thickness } \\
0.67 \mathrm{~mm}\end{array}$ & $\begin{array}{c}\text { Slice Thickness } \\
2 \mathrm{~mm}\end{array}$ \\
\hline Mean Volume $\left(\mathrm{cm}^{3}\right)$ & 50.03 & 50.09 \\
\hline $\mathrm{SD}\left(\mathrm{cm}^{3}\right)$ & 2.05 & 2.33 \\
\hline$p$-value & \multicolumn{2}{|c|}{0.950} \\
\hline & $\begin{array}{l}\text { PR }=0.5 \\
\text { overlap }\end{array}$ & $\begin{array}{c}\text { PR }=1 \\
\text { No overlap }\end{array}$ \\
\hline Mean Volume $\left(\mathrm{cm}^{3}\right)$ & 50.07 & 50.05 \\
\hline $\mathrm{SD}\left(\mathrm{cm}^{3}\right)$ & 2.11 & 2.28 \\
\hline$p$-value & \multicolumn{2}{|c|}{0.987} \\
\hline
\end{tabular}

Table 3. Results from preliminary assessment of EVAR-eaSE accuracy using radiological phantom 2.

\begin{tabular}{|c|c|c|}
\hline Phantom 2 & $\begin{array}{c}\text { Slice Thickness } \\
0.67 \mathrm{~mm}\end{array}$ & $\begin{array}{c}\text { Slice Thickness } \\
2 \mathrm{~mm}\end{array}$ \\
\hline Mean Volume $\left(\mathrm{cm}^{3}\right)$ & 107.18 & 107.94 \\
\hline $\mathrm{SD}\left(\mathrm{cm}^{3}\right)$ & 5.18 & 5.05 \\
\hline \multirow[t]{2}{*}{$p$-value } & \multicolumn{2}{|c|}{0.813} \\
\hline & $\begin{array}{l}\text { PR }=0.5 \\
\text { overlap }\end{array}$ & $\begin{array}{c}\text { PR }=1 \\
\text { No overlap }\end{array}$ \\
\hline Mean Volume $\left(\mathrm{cm}^{3}\right)$ & 106.41 & 107.09 \\
\hline $\mathrm{SD}\left(\mathrm{cm}^{3}\right)$ & 4.62 & 4.03 \\
\hline$p$-value & & \\
\hline
\end{tabular}

The Mean EVAR-eaSE volume (averaging results from processing all scans, regardless of different acquisition parameters) was $50.06 \pm 2.13 \mathrm{~cm}^{3}$, and $107.53 \pm 4.87 \mathrm{~cm}^{3}$ ) for phantom 1 and phantom 2 , respectively. When comparing these Mean calculated volumes with the real phantom volumes (Table 4), a statistically significant percentage difference was found for both phantoms $(-7.7 \%$ and $-2.5 \%$, respectively, $p<0.005)$. Particularly, the low percentage difference obtained for Phantom 2 demonstrated a good accuracy of EVAR-eaSE tool, being in line with the accuracy values reported in similar phantom evaluations for other software mainly intended for calculation of cerebral aneurysm volumes and packing densities [16,17]. 
Table 4. Comparative results between mean "computed" volume (by EVAR-eaSE) and "real" volume (by gauge), for both radiological phantoms.

\begin{tabular}{cccc}
\hline & $\begin{array}{c}\text { Computed Volume }\left[\mathrm{cm}^{3}\right] \\
\text { (by EVAR-eaSE) }\end{array}$ & $\begin{array}{c}\text { Real } \\
\text { Volume }\left[\mathrm{cm}^{3}\right] \\
\text { (by Gauge) }\end{array}$ & \% Difference \\
\hline Phantom 1 & 50.06 & 54.24 & $-7.7 \%$ \\
\hline Phantom 2 & 107.53 & 110.33 & $-2.5 \%$ \\
\hline
\end{tabular}

\subsection{Results of Comparative Analysis}

For the analyzed 26 AAA cases, Mean values of AAA-LV and AAA-TV obtained from EVAR-eaSE and the same volumes calculated using 3Mensio were significantly positively correlated $(r=0.940$, $\mathrm{r}=0.954, p<0.001)$. (Table 5).

Table 5. Paired samples correlation table between EVAR-eaSE and 3Mensio volumetric computations, for 26 retrospective abdominal aortic aneurysm (AAA) cases.

\begin{tabular}{|c|c|c|}
\hline \multicolumn{3}{|c|}{ AAA-Lumen Volume (LV) } \\
\hline & 3Mensio & EVAR-eaSE \\
\hline Mean $\left(\mathrm{cm}^{3}\right)$ & 89.73 & 85.38 \\
\hline $\operatorname{SD}\left(\mathrm{cm}^{3}\right)$ & 25.70 & 25.25 \\
\hline Correlation (r) & \multicolumn{2}{|c|}{0.940} \\
\hline$p$-value & \multicolumn{2}{|c|}{$<0.001$} \\
\hline \multicolumn{3}{|c|}{ AAA-Total Volume (TV) } \\
\hline & 3Mensio & EVAR-eaSE \\
\hline Mean TV $\left(\mathrm{cm}^{3}\right)$ & 142.19 & 127.96 \\
\hline $\mathrm{SD}\left(\mathrm{cm}^{3}\right)$ & 44.88 & 40.65 \\
\hline Correlation (r) & \multicolumn{2}{|c|}{0.954} \\
\hline$p$-value & \multicolumn{2}{|c|}{$<0.001$} \\
\hline
\end{tabular}

\section{Discussion and Conclusions}

In this paper, we have presented EVAR-eaSE software, a user-friendly tool that we have developed to ease the preoperative planning of EVAR procedure, particularly the AAA sac embolization (SE) using coils.

As key advantage, the tool provides semi-automatic segmentation of patient CTA scans and the computation of aneurysm volumes, thus allowing time saving for clinicians, as well as the reduction of operator-dependency in preoperative planning procedures. The software is easy-to-use after a brief training, without the need of specific programming knowledge.

Aneurysm volumes calculation is important to choose endovascular treatment modalities and to plan the optimal number of coils to be inserted for AAA SE. Many authors have previously reported the difficulty of determining true aneurysm volume [20-23]. As noted by Piotin et al. [21] and Sluzewski et al. [22], volume calculations based on 2-dimensional images can be adversely affected by the lack of a fiduciary marker and irregular aneurysm shapes, as well as simplistic spherical or elliptical geometric models can be inaccurate.

The developed EVAR-eaSE software provides segmentation, 3D modeling and automatic computation of the aneurysm volumes of interest for preoperative planning, starting from patient CTA scans. In the current version, the lumen volume segmentation is particularly efficient, since it may be achieved quickly and automatically with only two clicks by the user just selecting the first and the last slice in the patient scan. This result has been obtained following the example of previously reported 
approaches for automatic blood vessel segmentation from CTA $[24,25]$ that use a pre-processing stage for obtaining better segmentation results. In our case, a dedicated contrast-enhanced algorithm was implemented based on contrast stretching for remapping the image so it can occupy the whole gray shade spectrum. This specific feature helps the surgeon to better identify in the segmentation window the number of EPV of the aneurysm, which represents a predictive risk factor for ELIIp.

Comparing EVAR-eaSE with other commercial EVAR planning software or aneurysm volume calculators, the distinctive feature of our tool is the possibility to manage in an easy way the whole process of planning the SE during EVAR, this including: CTA patient scan segmentation and aneurysm volumes computation; setting of the endograft type and size for implant; computation of the endoluminal FV; and the optimal number of coils to be implanted. The software also provides calculation of a set of data useful for supporting EVAR planning (Table 1). A comparison of the EVAR-eaSE tool with other available software for aneurysm volume computation and SE planning, mainly in neurovascular field, is presented below in tabular format (Table 6).

Table 6. Comparative table of EVAR-eaSE with other available software tools.

\begin{tabular}{|c|c|c|c|c|c|}
\hline \multirow[b]{2}{*}{ Feature } & \multicolumn{5}{|c|}{ SOFTWARE TOOLS } \\
\hline & EVAR-eaSE & 3Mensio & AngioSuite & AngioCalc & Leonardo \\
\hline $\begin{array}{l}\text { Main field of } \\
\text { application }\end{array}$ & Vascular & Vascular & Neurovascular & $\begin{array}{c}\text { Neurovascular/ } \\
\text { Peripheral }\end{array}$ & Neurovascular \\
\hline $\begin{array}{l}\text { Imaging } \\
\text { source }\end{array}$ & CTA & CTA & Biplane angiograms & $\begin{array}{l}\text { Not applicable (use of } \\
\text { ellipsoid and } \\
\text { multi-lobular } \\
\text { geometric models for } \\
\text { the aneurysm) [16] }\end{array}$ & $\begin{array}{c}\text { 3D rotational } \\
\text { angiograms }\end{array}$ \\
\hline $\begin{array}{c}\text { Image } \\
\text { segmentation }\end{array}$ & $\begin{array}{c}\text { YES } \\
\text { (semi-automatic } \\
\text { segmentation } \\
\text { of AAA lumen } \\
\text { and AAA total } \\
\text { volume) }\end{array}$ & $\begin{array}{c}\text { YES } \\
\text { (segmentation } \\
\text { based on } \\
\text { manual } \\
\text { selection of } \\
\text { fiduciary } \\
\text { markers along } \\
\text { the aneurysm } \\
\text { shape) }[14,15]\end{array}$ & $\begin{array}{c}\text { YES } \\
\text { (segmentation based } \\
\text { on a calibration } \\
\text { performed from 2D } \\
\text { aneurysm } \\
\text { measurements, 3D } \\
\text { measurements and } \\
\text { fiduciary markers) } \\
\text { [17] }\end{array}$ & Not applicable & $\begin{array}{c}\text { YES } \\
\text { (delineation of } \\
\text { aneurysm } \\
\text { contour in } \\
\text { every slice in } \\
\text { axial views) } \\
\text { [16] }\end{array}$ \\
\hline $\begin{array}{l}\text { Computation } \\
\text { of aneurysm } \\
\text { volumes }\end{array}$ & YES & YES & YES & $\begin{array}{c}\text { YES } \\
\text { (shape model selection } \\
\text { and input of 2D } \\
\text { aneurysm } \\
\text { measurements) }\end{array}$ & YES \\
\hline $\begin{array}{c}\text { Input of } \\
\text { endograft } \\
\text { and/or coils } \\
\text { size }\end{array}$ & YES & NO & YES & YES & $\mathrm{NO}$ \\
\hline $\begin{array}{l}\text { Computation } \\
\text { of planning } \\
\text { parameters }\end{array}$ & $\begin{array}{c}\text { YES } \\
\text { (see Table 1) }\end{array}$ & $\begin{array}{l}\text { YES } \\
{[14,15]}\end{array}$ & $\begin{array}{c}\text { YES } \\
\text { (packing density) } \\
{[17]}\end{array}$ & $\begin{array}{c}\text { YES } \\
\text { (packing density) } \\
{[16,17]}\end{array}$ & $\mathrm{NO}$ \\
\hline
\end{tabular}

The commercial 3Mensio ${ }^{\mathrm{TM}}$ (Vascular Imaging, Bilthoven, The Netherlands) is a dedicated sizing software typically used for planning EVAR, treatment of thoracic aneurysms (TEVAR) or placement of fenestrated stents (FEVAR) [14,15]. Although it offers automatic vessel segmentation and center lumen line detection as well as the assessment of vessel tortuosity with respect to length measurements, the aneurysm volume calculation still remains quite time-consuming and operator-dependent, requiring a lot of "manual" interaction by the user, e.g., for the selection of fiduciary markers along the aneurysm shape on bi-dimensional images). Sometimes the marker selection has to be repeated for more than one $2 \mathrm{D}$ projection, and for each computation of the various aneurysm volumes of interest for preoperative planning (Figure 7). Moreover, the estimation of endoluminal FV available for SE with coils cannot be directly provided by the software, but the user has to calculate it offline by subtracting the approximated endograft volume to the calculated AAA lumen volume (Figure 7d). 


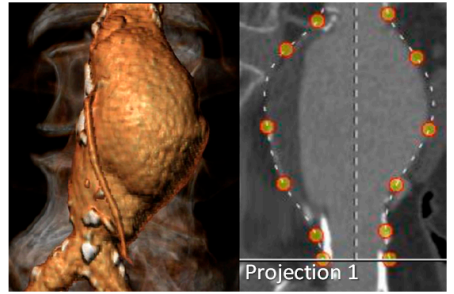

(a)

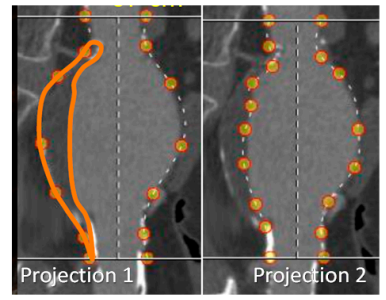

(b)

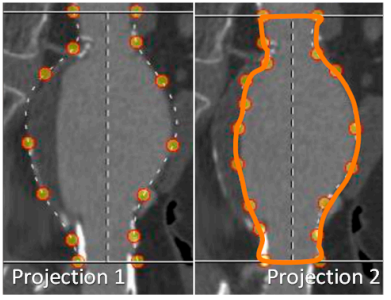

(c)

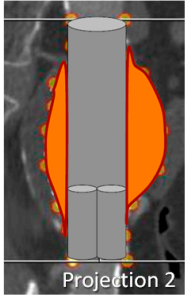

(d)

Figure 7. Example of consecutive steps for aneurysm volumes computation when using 3Mensio, based on manual selection of fiduciary markers along the vessel profile in two different planar projections: selection for abdominal aortic aneurysm (AAA) Total volume (a), AAA thrombus and lumen volumes $(\mathbf{b}, \mathbf{c})$. Calculation of endoluminal Free Volume (FV) available for Sac Embolization (SE) (orange area in (d)) is not directly provided by the software but has to be performed offline.

Vessel segmentation in 3Mensio strictly depends on manual selection of the fiduciary markers on the external sac profile, while EVAR-eaSE uses an automatic seeded region grow algorithm starting from a clicked seed voxel within the volume (Figure 8); this different approach may explain the small differences found for the two software in the computed volumes for the same AAA case (Table 5).

(a)

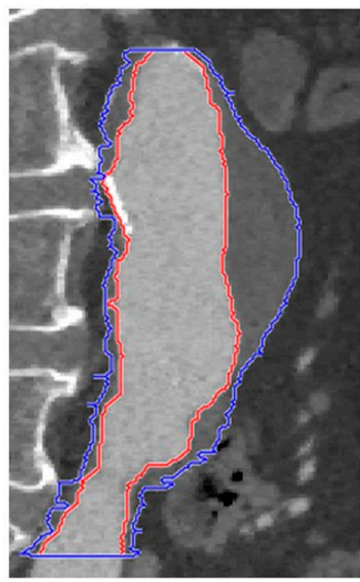

(b)

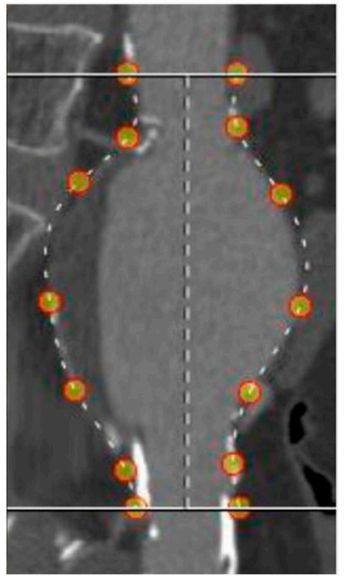

Figure 8. Example of aneurysm segmentation provided by: (a) EVAR-eaSE software using an automatic seeded region grow algorithm starting from inside; (b) 3Mensio requiring manual selection of markers on the external sac profile.

Among the other available software applications, mainly intended for neuro-interventionalists, the AngioCalc is an online calculator that estimates cerebral and peripheral aneurysm volume and percent packing volume after selection within a list of commercial neurovascular coils [16,17]. Its major limitation is in the use of ellipsoid and multi-lobular geometric models for approximating the aneurysm, that do not take into account the real irregularity of patient-specific aneurysm anatomies. Another system (AngioSuite, Cascade Medical, Knoxville, TN, USA) is able to calculate aneurysm volumes from biplane angiograms after calibration from known fiduciary markers, in a good accurate way [17]. Finally, with Leonardo workstation (Siemens, Erlangen, Germany) the aneurysm dimensions and volumes can be analyzed and computed starting from three-dimensional rotational angiograms windowed to the best visual appearance, but results are not particularly accurate [16,17].

EVAR-EaSE software achieves a good level of accuracy in 3D aneurysm volume reconstruction and calculation, as demonstrated by the performed tests on radiological phantoms. An interesting 
finding was also that the volumetric accuracy provided by EVAR-eaSE software does not depend on the CTA scan parameters.

The mean difference we found between the real and the segmented volume for phantom 2 was comparable to percentage difference $(2.2 \%)$ previously reported in a similar accuracy evaluation performed for AngioSuite software [17], considered the most accurate method to calculate aneurysm volume among various angiography-based and tomographic-based methods [16].

The slight volume underestimation obtained with EVAR-eaSE may depend on the phantom plastic material and the surrounding air that reduce the efficiency of the EVAR-eaSE contrast-enhanced algorithm, tuned for lumen vessel segmentation.

Study Limitations:

At present, the functionalities of EVAR-eaSE software are not fully optimized. In the current version, the AAA total volume segmentation may require subsequent interactions by the user to change and set the optimal parameters to improve the segmentation result. Future developments will be addressed to optimize this step, as well as to implement the calculation of additional parameters, such as the packing density, as already provided by neurovascular software tools [16].

The obtained preliminary results of good accuracy require to be confirmed on a wider range of volumes and phantom shapes more adherent to the real in vivo anatomy.

In the present study, we do not compare the EVAR-eaSE tool with 3Mensio in term of accuracy, but we only verify if there is a similar trend between the volumetric computations obtained by the two software tools (having already available 3Mensio data for the retrospective database of AAA cases treated with EVAR coil embolization). As next step, it would be interesting to evaluate and compare the accuracy between the new EVAR-eaSE tool and 3Mensio by performing the accuracy tests on phantoms also for 3Mensio.

The current study also lacks of a comparison of EVAR-eaSE results when the tool is used by different operators on the same patient scan. Future studies could be addressed to evaluate any possible inter-operator differences in terms of volumetric outputs and execution time.

Moreover, a feasibility study should be planned and conducted on EVAR-eaSE implementation and usability before promoting it for general use in the clinical practice.

In summary, the new EVAR-eaSE software provides an easy and accurate tool for calculating AAA volumes from patient-specific CTA scans, and for estimating the optimal number of coils to be implanted during SE to avoid long-term recurrences. The introduction of this software in routine preoperative planning may facilitate the practical use and acceptance of SE procedure during EVAR.

Supplementary Materials: The following are available online at http://www.mdpi.com/2076-3417/10/18/6252/s1, Video S1: EVAR-eaSE Demo application.

Author Contributions: Conceptualization, L.C., S.L. and E.M.; methodology, L.C., S.L., C.M.; software, S.L.; data analysis and curation, L.C., B.B.; writing-original draft preparation, L.C. and S.L.; writing-review and editing, L.C., S.L. and E.M.; supervision, M.G. and E.M. All authors have read and agreed to the published version of the manuscript.

Funding: This research received no external funding.

Conflicts of Interest: The authors declare no conflict of interest.

\section{References}

1. Patel, R.; Sweeting, M.; Powell, J. Endovascular versus open repair of abdominal aortic aneurysm in 15-years' follow-up of the UK endovascular aneurysm repair trial 1 (EVAR trial 1): A randomised controlled trail. J. Vasc. Surg. 2017, 65, 585. [CrossRef]

2. Pini, R.; Faggioli, G.; Longhi, M.; Mauro, R.; Freyrie, A.; Gargiulo, M.; Gallitto, E.; Mascoli, C.; Stella, A. The influence of study design on the evaluation of ruptured abdominal aortic aneurysm treatment. Ann. Vasc. Surg. 2014, 28, 1568-1580. [CrossRef] [PubMed] 
3. Jones, J.E.; Atkins, M.D.; Brewster, D.C.; Chung, T.K.; Kwolek, C.J.; Lamuraglia, G.M.; Hodgman, T.M.; Cambria, R.P. Persistent type 2 endoleak after endovascular repair of abdominal aortic aneurysm is associated with adverse late outcomes. J. Vasc. Surg. 2007, 46, 1-8. [CrossRef] [PubMed]

4. Abularrage, C.J.; Crawford, R.S.; Conrad, M.F.; Lee, H.; Kwolek, C.J.; Brewster, D.C.; Cambria, R.P.; Lamuraglia, G.M. Preoperative variables predict persistent type 2 endoleak after endovascular aneurysm repair. J. Vasc. Surg. 2010, 52, 19-24. [CrossRef] [PubMed]

5. Lo, R.C.; Buck, D.B.; Herrmann, J.; Hamdan, A.D.; Wyers, M.; Patel, V.I.; Fillinger, M.; Schermerhorn, M.L. Vascular Study Group of New England Risk factors and consequences of persistent type II endoleaks. J. Vasc. Surg. 2016, 63, 895-901. [CrossRef] [PubMed]

6. Piazza, M.; Squizzato, F.; Zavatta, M.; Menegolo, M.; Ricotta, J.J.; Lepidi, S.; Grego, F.; Antonello, M. Outcomes of endovascular aneurysm repair with contemporary volume-dependent sac embolization in patients at risk for type II endoleak. J. Vasc. Surg. 2016, 63, 32-38. [CrossRef] [PubMed]

7. Fabre, D.; Fadel, E.; Brenot, P.; Hamdi, S.; Caro, A.G.; Mussot, S.; Becquemin, J.-P.; Angel, C. Type II endoleak prevention with coil embolization during endovascular aneurysm repair in high-risk patients. J. Vasc. Surg. 2015, 62, 1-7. [CrossRef] [PubMed]

8. Dosluoglu, H.H.; Rivero, M.; Khan, S.Z.; Cherr, G.S.; Harris, L.M.; Dryjski, M.L. Pre-emptive nonselective perigraft aortic sac embolization with coils to prevent type II endoleak after endovascular aneurysm repair. J. Vasc. Surg. 2019, 69, 1736-1746. [CrossRef] [PubMed]

9. Mascoli, C.; Freyrie, A.; Gargiulo, M.; Gallitto, E.; Pini, R.; Faggioli, G.; Serra, C.; De Molo, C.; Stella, A. Selective Intra-procedural AAA sac Embolization During EVAR Reduces the Rate of Type II Endoleak. Eur. J. Vasc. Endovasc. Surg. 2016, 51, 632-639. [CrossRef]

10. Otsu, M.; Ishizaka, T.; Watanabe, M.; Hori, T.; Kohno, H.; Ishida, K.; Nakaya, M.; Matsumiya, G. Analysis of anatomical risk factors for persistent type II endoleaks following endovascular abdominal aortic aneurysm repair using CT angiography. Surg. Today 2015, 46, 48-55. [CrossRef]

11. Gallitto, E.; Gargiulo, M.; Mascoli, C.; Freyrie, A.; De Matteis, M.; Serra, C.; Massoni, C.B.; Faggioli, G.; Stella, A. Persistent type II endoleak after EVAR: The predictive value of the AAA thrombus volume. J. Cardiovasc. Surg. 2015, 59, 79-86.

12. Aoki, A.; Maruta, K.; Hosaka, N.; Omoto, T.; Masuda, T.; Gokan, T. Evaluation and coil embolization of the aortic side branches for prevention of type ii endoleak after endovascular repair of abdominal aortic aneurysm. Ann. Vasc. Dis. 2017, 10, 351-358. [CrossRef] [PubMed]

13. Mascoli, C.; Gargiulo, M.; Gallitto, E.; Longhi, M.; Pini, R.; Ancetti, S.; Faggioli, G.; Stella, A. IP071. Persistent type ii endoleak prevention by abdominal aortic aneurysm sac embolization during EVAR: An analysis of aneurysm volume and coils concentration. J. Vasc. Surg. 2017, 65, 75S-76S. [CrossRef]

14. Reimerink, J.J.; Marquering, H.A.; Vahl, A.; Wisselink, W.; Schreve, M.A.; De Boo, D.W.; Reekers, J.A.; Legemate, D.A.; Balm, R. Semiautomatic sizing software in emergency endovascular aneurysm repair for ruptured abdominal aortic aneurysms. Cardiovasc. Interv. Radiol. 2013, 37, 623-630. [CrossRef]

15. Van Keulen, J.W.; Moll, F.L.; Tolenaar, J.L.; Verhagen, H.J.M.; Van Herwaarden, J.A. Validation of a new standardized method to measure proximal aneurysm neck angulation. J. Vasc. Surg. 2010, 51, 821-828. [CrossRef]

16. La Garma, V.H.E.-D.; Zenteno, M.; Padilla-Vázquez, F.; San-Juan, D.; Cerón-Morales, A. Comparative analysis of aneurysm volume by different methods based on angiography and computed tomography angiography. Neurosurg. Rev. 2018, 41, 1013-1019. [CrossRef]

17. Woodward, K.; Forsberg, D.A. AngioSuite: An accurate method to calculate aneurysm volumes and packing densities. J. NeuroInterv. Surg. 2012, 5, 28-32. [CrossRef]

18. Chan, T.F.; Vese, L. Active contours without edges. IEEE Trans. Image Process. 2001, 10, 266-277. [CrossRef]

19. Oliveira, R.; Filho, M.E.; Ma, Z.; Papa, J.P.; Pereira, A.S.; Tavares, J.M.R. Computational methods for the image segmentation of pigmented skin lesions: A review. Comput. Methods Programs Biomed. 2016, 131, 127-141. [CrossRef]

20. Bescós, J.O.; Slob, M.J.; Slump, C.H.; Sluzewski, M.; Van Rooij, W. Volume measurement of intracranial aneurysms from 3D rotational angiography: Improvement of accuracy by gradient edge detection. Am. J. Neuroradiol. 2005, 26, 2569-2572. 
21. Piotin, M.; Daghman, B.; Mounayer, C.; Spelle, L.; Moret, J. Ellipsoid approximation versus 3D rotational angiography in the volumetric assessment of intracranial aneurysms. Am. J. Neuroradiol. 2006, 27, 839-842. [PubMed]

22. Sluzewski, M.; Van Rooij, W.J.; Slob, M.J.; Bescós, J.O.; Slump, C.H.; Wijnalda, D. Relation between Aneurysm Volume, Packing, and Compaction in 145 Cerebral Aneurysms Treated with Coils. Radiology 2004, 231, 653-658. [CrossRef] [PubMed]

23. Sadato, A.; Hayakawa, M.; Tanaka, T.; Hirose, Y. Comparison of cerebral aneurysm volumes as determined by digitally measured 3D rotational angiography and approximation from three diameters. Interv. Neuroradiol. 2011, 17, 154-158. [CrossRef] [PubMed]

24. Bozkurt, F.; Köse, C.; Sarı, A. An inverse approach for automatic segmentation of carotid and vertebral arteries in CTA. Expert Syst. Appl. 2018, 93, 358-375. [CrossRef]

25. Moccia, S.; De Momi, E.; El Hadji, S.; Mattos, L.S. Blood vessel segmentation algorithms—Review of methods, datasets and evaluation metrics. Comput. Methods Progr. Biomed. 2018, 158, 71-91. [CrossRef]

(C) 2020 by the authors. Licensee MDPI, Basel, Switzerland. This article is an open access article distributed under the terms and conditions of the Creative Commons Attribution (CC BY) license (http://creativecommons.org/licenses/by/4.0/). 